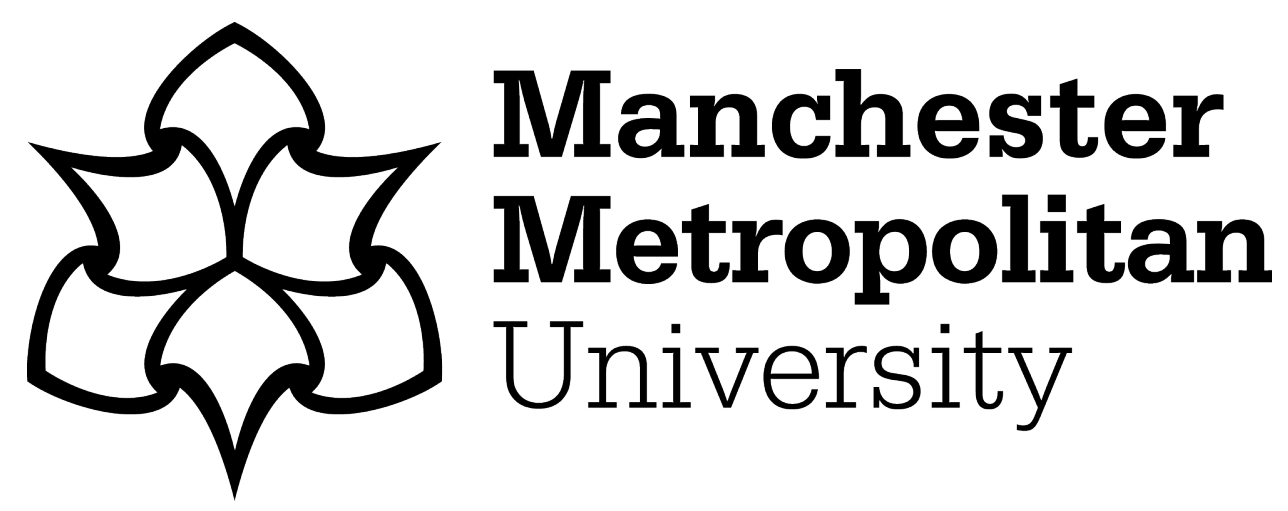

Sims, DT, Burden, A, Payton, C, Onambélé-Pearson, GL and Morse, CI (2018) A quantitative description of self-selected walking in adults with Achondroplasia using the gait profile score. Gait \& Posture, 68. pp. 150154. ISSN 0966-6362

Downloaded from: https://e-space.mmu.ac.uk/621927/

Version: Published Version

Publisher: Elsevier

DOI: https://doi.org/10.1016/j.gaitpost.2018.11.019

Usage rights: Creative Commons: Attribution-Noncommercial-No Derivative Works 4.0

Please cite the published version 
Full length article

\title{
A quantitative description of self-selected walking in adults with Achondroplasia using the gait profile score
}

\author{
D.T. Sims*, A. Burden, C. Payton, G.L. Onambélé-Pearson, C.I. Morse \\ Health, Exercise and Active Living Research, Manchester Metropolitan University, United Kingdom
}

\section{A R T I C L E I N F O}

\section{Keywords:}

Achondroplasia

Walking

Gait profile score

Movement analysis profile

Gait kinematics

\begin{abstract}
A B S T R A C T
Background: Achondroplasia is characterised by a shorter appendicular limb-to-torso ratio, compared to age matched individuals of average stature (controls). Previous work shows gait kinematics of individuals with Achondroplasia differing to controls, but no global quantification of gait has been made in adults with Achondroplasia.

Aim: The aim of this study was to quantify gait differences between a group of adult males with Achondroplasia and controls during self-selected walking (SSW) using the Gait Profile Score (GPS).

Design: Whole body motion analysis of 10 adults with Achondroplasia ( $22 \pm 3 \mathrm{yrs}$ ) who had not undergone leg lengthening and 17 adult controls ( $22 \pm 2$ yrs) was undertaken using a 14 camera VICON system (100 Hz). For each group, fifteen root mean squared Gait Variable Scores (GVS, units ${ }^{\circ}$ ) were computed from lower limb kinematic data and then summed to calculate GPS ( ${ }^{\circ}$ ).

Results: The group with Achondroplasia had higher GVSs than controls in 10 of the 15 measures $(\mathrm{P}<0.05)$ with the largest differences found in ankle plantar/dorsiflexion $(\mathrm{P}<0.001)$, knee flexion/extension $(\mathrm{P}<0.001)$, and hip internal/external rotation $(\mathrm{P}<0.001)$. The GPS value of the group with Achondroplasia was $64 \%$ higher than controls $\left(11.4^{\circ}(2.0) \mathrm{v} 4.1^{\circ}(1.8), \mathrm{P}<0.001\right)$.

Conclusion: Gait is quantitatively different in adults with Achondroplasia compared to controls. The differences in GPS between groups are due to differences in joint kinematics, which are possibly manifested by maintaining toe-clearance during swing. Gait models derived from the anatomy of individuals with Achondroplasia may improve these data.
\end{abstract}

\section{Introduction}

Achondroplasia is a genetic condition characterised by short stature due to a shorter growth of long bones in the appendicular skeleton compared to the torso. This presents as a disproportionate limb-to-torso length compared age matched able bodied individuals (controls) [1-3]. The shorter, disproportionate, leg length in individuals with Achondroplasia appear to alter gait kinematics at self-selected walking speeds (SSW) compared to controls [4-7]. Descriptions of gait in adults with Achondroplasia are sparse but do show that their knee and ankle are more flexed and dorsiflexed than controls during the whole gait cycle. These joint positions are possibly due to a larger foot-to-leg length ratio in individuals with Achondroplasia compared to controls. This relatively longer foot may require lower limb joints to be more flexed to avoid toe contact with the floor during swing [5]. Consequently, gait of individuals with Achondroplasia appears different to controls. With the large number of kinematic variables that are collected during gait however, quantifying any population's global difference to controls is difficult. In order to compare global gait scores for clinical populations to control populations, methods have been developed by incorporating a number of different kinematic variables. One such method is the Gait Profile Score (GPS); lower GPS values represent a more comparable movement pattern to that of a control population over a complete stride [8].

GPS has been used to compare differences in gait between individuals with and without gait limiting pathologies and has been conducted under various conditions [8-13]. It is derived by summing 15 root mean square (RMS) differences in gait related kinematics during the gait cycle. GPS correlates well with clinical assessments [10], has high face validity [9] and affords the ability to perform more powerful statistical analyses than other global gait indices, such as the Gait Deviation Index [14], thus allowing for a better understanding of clinical relevance [15]. Calculation of GPS allows for inter- and intrajoint and plane comparisons within and between groups with different

\footnotetext{
* Corresponding author at: Department of Exercise and Sport Science, Manchester Metropolitan University, Cheshire, CW1 5DU, United Kingdom.

E-mail address: d.sims@mmu.ac.uk (D.T. Sims).
} 
Table 1

Anthropometric values for the group with Achondroplasia and controls. Values given as mean (SD).

\begin{tabular}{|c|c|c|c|}
\hline & Achondroplasia & & Control \\
\hline Age (yrs) & $22(3)$ & & $22(2)$ \\
\hline Stature (m) & $1.38(0.05)$ & * & $1.79(0.08)$ \\
\hline Mass (kg) & $61.8(8.5)$ & * & $78.3(10.7)$ \\
\hline Leg Length (m) & $0.51(0.03)$ & * & $0.86(0.05)$ \\
\hline Foot Length (m) & $0.18(0.01)$ & * & $0.22(0.01)$ \\
\hline Relative Foot Length (\%) & $36.0(2.1)$ & * & $25.6(1.5)$ \\
\hline $\mathrm{SSW}\left(\mathrm{m} \cdot \mathrm{s}^{-1}\right)$ & $1.05(0.17)$ & * & $1.38(0.19)$ \\
\hline
\end{tabular}

SSW, Self-selected Walking Speed; * $\mathrm{P} \leq 0.001$.

pathologies. This is useful to determine which are the predominant joints affecting differences in gait between groups and therefore aid in gait rehabilitation or gait improvement interventions. For example, previous observations of a more flexed knee and dorsiflexed ankle throughout the stride of an individual with Achondroplasia would be highlighted and then combined with other joint kinematics to form a GPS [5]. Quantifying a global gait score for an individual with Achondroplasia would also provide reference data to monitor gait improvements following interventions, such as gait following leg-lengthening surgery $[4,7]$. However, to the authors' knowledge, no such measure of gait difference has been made in any population with Achondroplasia. The aim of this study was to describe the global SSW gait kinematics of adults with Achondroplasia using GPS and compare to controls.

\section{Method}

After providing written consent, 10 adults medically defined as presenting Achondroplasia, and 17 age matched controls volunteered to participate in the study (anthropometrics are given in Table 1). All participants self-reported as undertaking between 2-3 h of structured physical activity per week and were free from any gait limiting lower limb injury. None of the participants with Achondroplasia had undergone leg lengthened surgery. Ethical approval was obtained from the local committee and each participant attended one testing session in the biomechanics laboratory of Manchester Metropolitan University where anthropometric and kinematic assessments at SSW were conducted. Leg length $(\mathrm{m})$ of all participants was measured as the distance from the anterior iliac spine to the medial malleolus of the ankle while standing. Foot length (m) was measured as the vector distance between heel and toe markers from the biomechanical gait model described below. Relative foot length (\%) was then calculated as the ratio of foot and leg length, as done elsewhere [5].

\subsection{Self-selected walking speed and kinematic measures}

Three-dimensional (3D) motion analysis hardware (VICON, Oxford, UK) was used to determine gait parameters. Measures of leg length, knee width, ankle width and inter-ASIS distance of each participant were taken and entered into the software (Nexus 2.5) to estimate joint centre locations [16]. A conventional gait model (Plug-in-Gait) consisting of 39 markers was used to obtain lower limb joint kinematics and centre of mass kinematics by using inertial properties described by Dempster [17]. Fourteen cameras (VICON MX T160, $100 \mathrm{~Hz}$ ), calibrated following the manufacturer's guidelines, were positioned on scaffolding giving a $\sim 170 \mathrm{~m}^{3}$ capture area. Participants were invited to walk at a self-prescribed speed around the laboratory ( $\sim 40 \mathrm{~m}$ total) and then along $\sim 10 \mathrm{~m}$ walkway three times. Each time, a single stride of the left leg, nearest to the centre of the calibration volume, was used to determine lower limb and centre of mass kinematics of each participant. A Bonita camera $(720 \mathrm{C}, 100 \mathrm{~Hz})$ was placed perpendicular to the plane of motion and synchronised with the VICON system. Gait events were determined manually post-trial by using the video images provided by the Bonita camera. In all trials, kinematic data was smoothed using a Butterworth filter with a cut off frequency chosen based on residual analysis of each trail and then time normalised to 100 data points using a cubic spline interpolation method (Microsoft Excel macro, 2000). Lower limb and centre of mass kinematics were averaged for each participant and SSW was determined as the average speed of the centre of mass for the three strides.

\subsection{Gait profile score}

Based on the method proposed by Baker et al. [8] and presented in Baker et al. [10], 15 gait specific RMS differences, known as Gait Variable Scores (GVSs, units ${ }^{\circ}$ ) were calculated for each group. Specifically, GVSs were calculated for: pelvic tilt, obliquity and rotation; hip flexion/extension, abduction/adduction and internal/external rotation; knee flexion/extension; ankle plantar/dorsiflexion; foot progression; and, a total GVS for each leg (Eq. (1) [10],). For the GVSs of the adults with Achondroplasia, RMS differences were compared to the control mean, whereas the control group's GVSs were compared to their own mean. GPS (units ${ }^{\circ}$ ) was then calculated as the sum of the RMS of each groups' 15 GVSs, given in Eq. (2) [10].

$$
\begin{aligned}
G V S_{i} & =\sqrt{\frac{1}{T} \sum_{t=1}^{T}\left(x_{i, t}-\bar{x}_{i, t}^{r e f}\right)^{2}} \\
G P S & =\sqrt{\frac{1}{N} \sum_{i=1}^{N} G V S_{i}^{2}}
\end{aligned}
$$

Where $G V S_{i}$ is the $i^{\text {th }}$ gait variable score for a specified joint, $x_{i, t}$ is each participant's gait variable (Achondroplasia and control), $i$, calculated at a specific time point, $t$, and $\bar{x}_{i, t}^{r e f}$ is the same variable averaged from the reference group (control only), $T$ is the number of data points the gait cycle is divided into.

\subsection{Statistical analysis}

Each GVS was inferentially compared between groups and presented graphically as a collection of RMS differences known as the Movement Analysis Profile (MAP) [8]. All statistical analysis was conducted using SPSS (IBM, v24). An independent $t$-test was run between groups' anthropometric variables. A log-transformation of GVS was performed due to the skewed distribution of the data. To account for Type I errors, Mann Whitney-U tests were conducted to identify effects between groups' individual GVSs and GPS. Wilcoxon tests were performed within each group to identify effects between left and right legs for each GVS. Alpha was set to $\leq 0.05$, with data presented as median (interquartile range, IQR).

\section{Results}

There was no difference in age between groups $(P=0.487)$, while the group of adults with Achondroplasia were $23 \%$ shorter $(\mathrm{P}<0.001)$ and $19 \%$ lighter than controls $(\mathrm{P}<0.001$, Table 1$)$. The adults with Achondroplasia had a shorter leg $(\mathrm{P}<0.001)$ and foot $(\mathrm{P}<0.001)$ but had a longer foot-to-leg length ratio compared to controls $(\mathrm{P}<0.001$, Table 1). The adults with Achondroplasia were $24 \%$ slower than controls $(\mathrm{P}<0.001$, Table 1$)$.

No differences were found in any GVS score between the left and right leg within each group ( $\mathrm{P}>0.05$, Fig. 1 ). There was also no difference in pelvic anterior/posterior tilt GVS $(P=0.303)$, hip flexion/ extension GVS $(P=0.497)$ or foot progression GVS between groups $(\mathrm{P}=0.507$, Fig. 1$)$. The adults with Achondroplasia had a higher GVS in knee flexion/extension ( $\mathrm{P}<0.001$ ), ankle plantar/dorsiflexion $(\mathrm{P}<0.001)$, pelvis obliquity $(\mathrm{P}<0.001)$, hip abduction/adduction $(P=0.001)$, pelvis rotation $(P=0.006)$, hip rotation $(P=0.001)$ and 


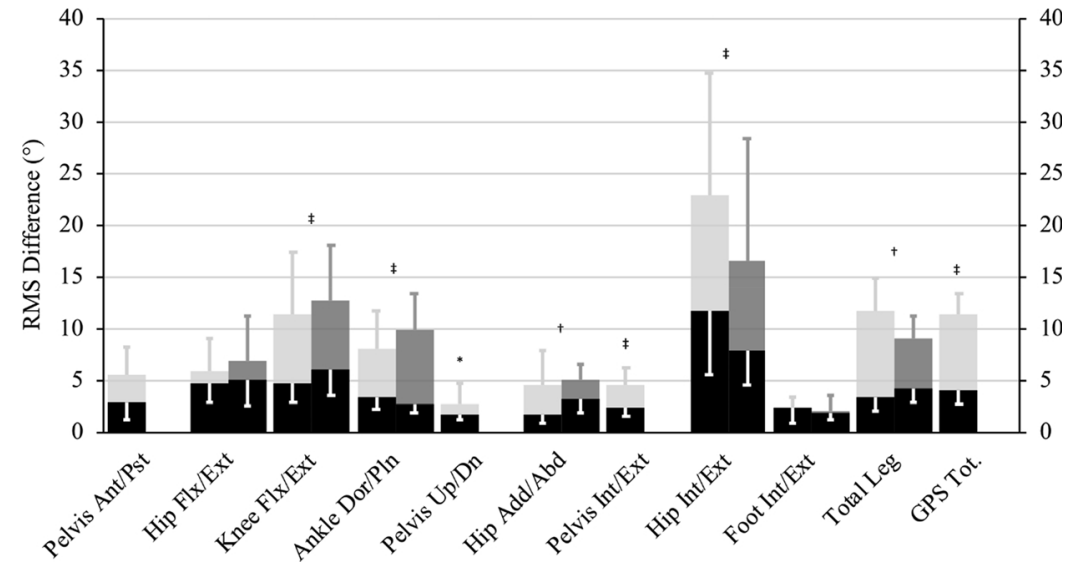

Fig. 1. Movement analysis profile of the group with Achondroplasia (grey) and controls (black) for one stride. Individual gait variable scores (GVS) for the left (light grey) and right (dark grey) sides. Note that the pelvis is one segment and therefore is presented for the left side only. Total GVS for the left and right leg and the gait profile score are presented on the rightmost columns. Data are presented as median and interquartile ranges. ${ }^{*} \mathrm{P}<0.05,{ }^{\dagger} \mathrm{P} \leq 0.01,{ }^{*} \mathrm{P} \leq 0.001$ for between group comparisons only. total leg GVS compared to controls $(P=0.020$, Fig. 1$)$. The group of adults with Achondroplasia also had a $64 \%$ greater total GPS than controls ( $\mathrm{P}<0.001$, Fig. 1).

\section{Discussion}

This study aimed to describe the SSW gait of adult males with Achondroplasia using GPS. The main findings were that 1) adults with Achondroplasia presented a number of higher GVSs, other than pelvis anterior/posterior tilt, hip flexion/extension and foot progression; and 2) GPS was $64 \%$ greater in the adults with Achondroplasia than controls, suggesting that the gait of adults with Achondroplasia is different to controls.

The GPS was devised to describe numerous gait related variables throughout the gait cycle using a single number. GPS has been used to describe and determine gait 'quality' in clinical populations compared to controls [8-10]. The results presented in this study suggest that the gait of adults with Achondroplasia is of a lower quality than controls. The GPS of the group with Achondroplasia $\left(11.4^{\circ}(2.0)\right)$ is consistent with conditions that present musculoskeletal impairments such as spina bifida, ligamentous laxity, paraplegia and Cerebral Palsy (7.5-14.5 $[10,13])$. While the GPS of the group with Achondroplasia is higher than controls, the term 'quality', that has been associated with GPS, is ambiguous in the current context as it has limited biomechanical or physiological meaning. Certainly, a higher GPS infers a difference in gait, but not necessarily a lower quality of gait; this is likely the case in many populations of anatomical deformity. Within this context, GPS would be strengthened alongside single joint kinematic patterns during the gait cycle, such as that applied in the present study (Fig. 2). While not presenting kinematic patterns of single joints, the GPS does provide a quantitative value of the RMS difference for individual joint planes (here as GVSs), unlike other global gait indices ((e.g. the Gait Deviation Index [14]). In the current study, all GVSs (other than pelvis anterior/ posterior tilt, hip flexion/extension and foot progression) were larger in the group with Achondroplasia. Of note, the GVSs of knee flexion/extension (55\%), ankle planta/dorsiflexion (64\%) and hip internal/external rotation $(50 \%)$ were the largest differences. The anthropometry and muscular strength of the adults with Achondroplasia as well as the methods used to obtain joint kinematics may explain the differences in hip, knee and ankle GVSs and GPS between groups.

The current group with Achondroplasia had a relatively longer foot (foot-to-leg length ratio) than controls, consistent with previous reports [5]. For the toe of the relatively longer foot to clear the floor during swing phase, more flexion of the lower limb joints is required. Indeed, more knee flexion and more ankle dorsiflexion were observed in the adults with Achondroplasia during gait (Fig. 2). These kinematic differences in the knee and ankle contribute to a higher knee flexion/extension GVS and ankle plantar/dorsiflexion GVS respectively. Both
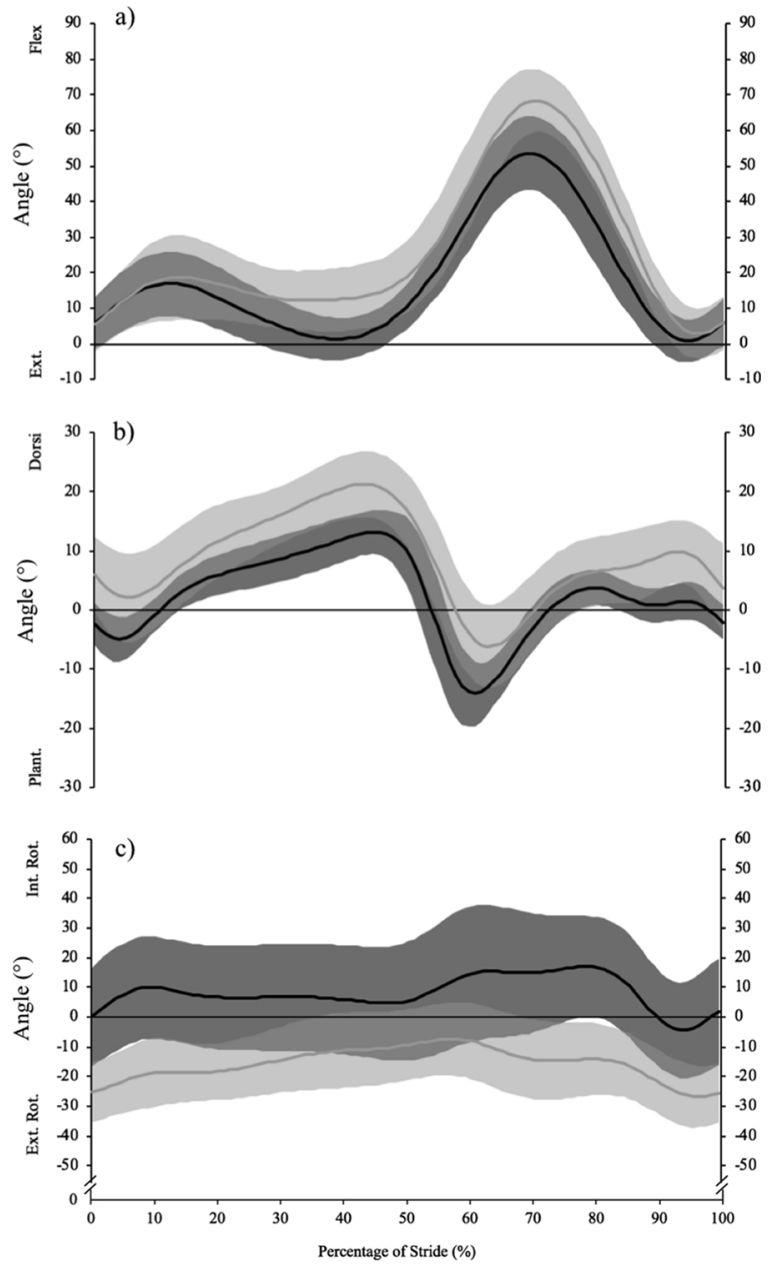

Fig. 2. Sagittal joint kinematics of a) knee and b) ankle and transverse kinematic patterns of c) hip during one complete stride for the group with Achondroplasia (light grey) and control group (dark grey). Solid lines represent the respective group mean and shaded areas represent \pm 1 standard deviation.

would contribute to a higher GPS in the group with Achondroplasia than controls, leading to the assumption of lower gait quality. Greater knee flexion and ankle dorsiflexion during gait is observed in the current group with Achondroplasia and in other groups with Achondroplasia compared to controls [5-7]. Differences are however observed in kinematic patterns of the current group with Achondroplasia compared to previous groups with Achondroplasia [5-7]. For example, a leg lengthened group, of similar age to the current cohort, appear less 
flexed at the knee and ankle [7]; this is likely due to a smaller foot-toleg length ratio in the leg lengthened group. Regardless of reported differences in gait between groups with Achondroplasia (e.g. age and leg length), it is likely that a GPS of any individual with Achondroplasia is higher than age matched controls due to the foot-to-leg length ratio. This topic though, requires further work in the population.

The higher knee flexion/extension GVS in current group of adults with Achondroplasia compared to controls could have been inflated by the joint marker placement and/or hip joint centre (HJC) predictions. The current study used a Plug-in-Gait model to determine joint kinematics. The model is hierarchal in that distal joints are predicted based on proximal joints. As such, the accurate prediction of the HJC is of utmost importance. The HJC prediction is based on leg length and the distance between the anterior and posterior processes of the iliac spine [16], which are disproportionate between the observed groups. While Plug-in-Gait does not use a direct HJC marker, an interpretation of the HJC (i.e. greater trochanter) is required to place the lateral thigh marker. The thigh marker is aligned between the identifiable greater trochanter and knee marker for both groups so that it is placed in the transverse plane of the femur. Separate analysis of the HJC in the current group with Achondroplasia group suggests that either the palpable identification of the greater trochanter does not represent the HJC or, the anthropometric dimensions used to predict HJC are incorrect for populations with Achondroplasia (Fig. 3). In the current group with Achondroplasia, the HJC appears to be more posterior than the identification of the greater trochanter which is contrary to previous work that has estimated the HJC of individuals with Achondroplasia. Broström et al. [18] showed that a functional method [19] of identifying HJC in individuals with Achondroplasia was $15.6 \mathrm{~mm}$ more posterior than the Plug-in-Gait model [16], but could range from 1.7 to $31.3 \mathrm{~mm}$ more posterior than Plug-in-Gait's prediction. Broström et al. suggested that a functional prediction of HJC in individuals with Achondroplasia should be incorporated into future gait research of individuals with Achondroplasia. However, were the functional model to be used in the present study, the HJC would more even more posterior in the group with Achondroplasia than that predicted here by Plug-inGait (Fig. 3). This would lead to greater knee flexion estimates of the group with Achondroplasia and in turn undoubtedly raise their respective GVSs and GPS. Conversely though, a more anteriorly placed thigh marker would result in greater internal hip rotation in either group [20]. The kinematic pattern of the hip in the group with Achondroplasia however, show a persistently more externally rotated hip than controls during the entire stride.

The higher hip internal/external GVS of the group with Achondroplasia is due to a more externally rotated hip. In controls, a larger beta-angle of the femur correlates positively with internal rotation of the hip [21]; a smaller beta-angle is observed in individuals with Achondroplasia though [22]. The smaller beta-angle observed in this population would therefore be a viable explanation for their more externally rotated hip during gait and their higher internal/external GVS than controls. No correlations between hip rotation angles and betaangles have been made in individuals with Achondroplasia though and is required to confirm this explanation. We have recently shown that a strength imbalance exists between the knee extensors and flexors in individuals with Achondroplasia [23]. Such an imbalance may exist in other muscle groups within the population, such as between the internal and external hip rotators. As strength correlates positively with muscle size [24,25], and with the external rotators of the hip being larger than the internal rotators [23], the potential imbalance may contribute to a more externally rotated hip. Again though, further work is necessary to demonstrate this explanation.

Biomechanical models used for the measurement of gait kinematics are developed by the joint markers being placed on anatomical locations which are an estimated distance from the joint centre. These distances are becoming more accurate through the use of imaging techniques such as magnetic resonance imaging [26] and computed tomography [27]. In contrast, the placement of kinematic markers on the group with Achondroplasia were based on the same anatomical palpitation designed to accurately predict joint centres in controls (see limitations section below). There is the potential therefore, that the process by which markers are placed on the individuals with Achondroplasia could induce systematic bias within some of the kinematic measures, e.g. knee flexion angles due to the misplacement of the thigh marker. Were there systematic bias in marker placement though, the direction of the difference between groups' knee flexion, ankle dorsiflexion and external hip rotation positions would likely be the same. In fact, the externally rotated position of the hips of the group with Achondroplasia may be greater than that reported in the current study due to the above arguments regarding beta-angle of the femur in individuals with Achondroplasia compared to controls. We are confident however, that regardless of the model used, or suggested for gait analyses in individuals with Achondroplasia [18,19], only knee flexion/ extension and hip rotation GVSs would be affected in this group were the thigh marker placement incorrect. It is probable therefore, that the GPS of any individual with Achondroplasia (e.g. child or following gait correction interventions) would be higher than controls and therefore the use of GPS to compare gait of individuals with Achondroplasia to controls is somewhat null. A GPS analysis of an individual with Achondroplasia would certainly be worthwhile when compared to other groups with Achondroplasia, for example following exercise or leg lengthening surgery. We suggest therefore, that the data presented here act as reference data for future individuals with Achondroplasia to be compared to, rather than indicate 'gait corrections' to lower the GPS of the group with Achondroplasia to be in line with controls.

Limitations

Despite what appears to be a relatively low sample, the group of

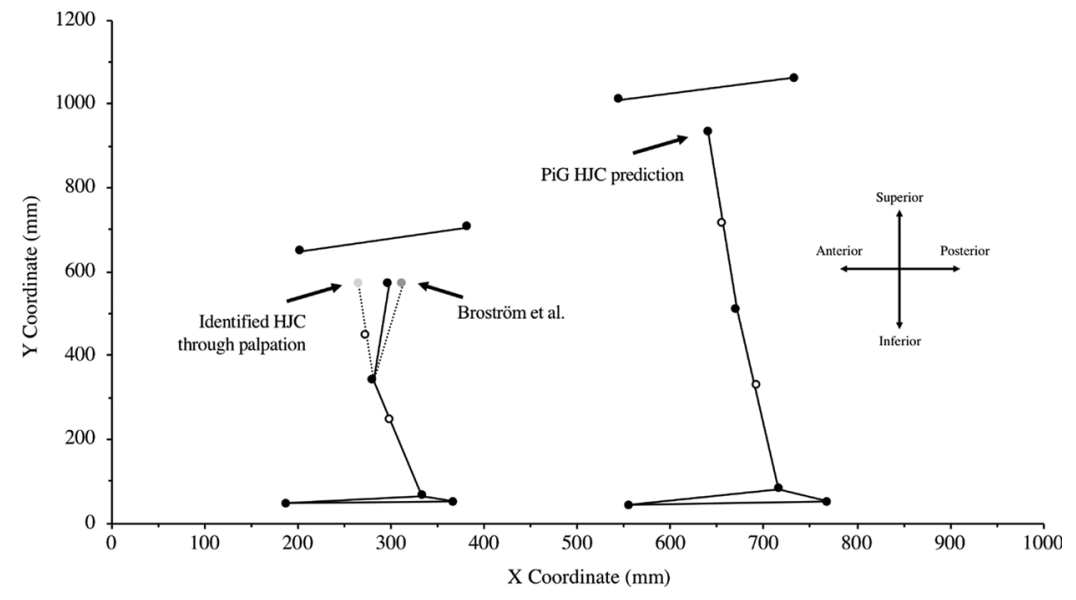

Fig. 3. Sagittal view of the mean position Plug-in-Gait markers for the group with Achondroplasia (left) and controls (right). Black circles represent the respective bony landmarks and the hip joint centre (HJC) while white circles represent segment markers used for rotational variables in the Plug-in-Gait model (PiG). For the example of individuals with Achondroplasia (left), the dark grey circles represent the estimated HJC from the equations presented by Broström et al. [19] and the light grey circles represent the estimated HJC based on the palpable greater trochanter, which in this case is extrapolated from the knee and thigh marker. Solid lines represent respective segments, from which joint angles are calculated and dotted line represents the estimated thigh segments based on the HJC prediction from Broström et al. [19] and the palpable greater trochanter. 
individuals with Achondroplasia included in this study consisted of $\sim 13 \%$ of the registered U.K. population of physically active males between the age of 18-35 yrs. The current group with Achondroplasia also represents the most homogenous skeletal dysplasic group available in the gait literature (i.e. accounting for age, sex, condition, pre-leg lengthened and activity levels) and does provide a useful reference for other individuals with Achondroplasia to be compared to due to the homogeneity of our group. However, while the data from this study are applicable to the other active males with Achondroplasia within the U.K., and the world, they do not necessarily represent other cohorts with Achondroplasia, such as females, the elderly, children or inactive males.

With the Plug-in-Gait model being developed in controls, the control data presented in this study is representative of the wider control data. Indeed, the controls' GPS data matches that of other control GPS data available in the literature (e.g. $[8,9,28]$ ). Given the arguments within the discussion that there may be systematic error in the placement of the thigh marker in the group with Achondroplasia, further work should ideally develop gait models for these individuals through the use of detailed imaging techniques, like those available in control groups and children $[26,27]$. Furthermore, a full spatio-temporal analysis of gait that incorporates other gait related biomechanical measures, such as ground reaction forces or electromyography data, is required to fully identify the mechanisms to why the gait of individuals with Achondroplasia is different to controls.

\section{Conclusion}

The current study aimed to describe the SSW gait of an adult group with Achondroplasia using GPS. While we demonstrate that the GPS of individuals with Achondroplasia is $64 \%$ higher than controls, this is most likely due to higher knee flexion/extension, ankle plantar/dorsiflexion and hip rotation GVSs in the group with Achondroplasia. The difference in GPS is most likely due to the need to avoid toe contact during swing phase of the group with Achondroplasia.

\section{Conflict of interest}

The authors have no conflict of interest.

\section{Acknowledgements}

The authors would like to thank the Dwarf Sports Association (Dronfield, UK. Registered Charity number 1041961) for their assistance in recruitment, Dream it Believe it Achieve it (Northwich, UK. Registered Charity Number: 1153116) from which D. Sims received initial postgraduate funding and Raisa Rossignaud for her efforts during data collection.

\section{References}

[1] W.A. Horton, J.G. Hall, J.T. Hecht, Achondroplasia, Lancet 370 (2007) 162-172.
[2] A. Superti-Furga, S. Unger, Nosology and classification of genetic skeletal disorders: 2006 revision, Am. J. Med. Genet. A 143A (2007) 1-18.

[3] D. Krakow, D.L. Rimoin, The skeletal dysplasias, Genet. Med. 12 (2010) 327-341.

[4] S. Rethlefsen, V.T. Tolo, Gait analysis before and after tibial osteotomy in achondroplasia, Gait Posture 7 (1998) 174-175.

[5] R. Egginton, C. Newman, M. Walsh, A. Jenkinson, D. Bennett, T. O’Brien, Kinematic characteristics of achondroplasia, Gait Posture 24 (2006) S249-S250.

[6] M. Inan, M. Thacker, C. Church, F. Miller, W.G. Mackenzie, D. Conklin, Dynamic lower extremity alignment in children with achondroplasia, J. Pediatr. Orthop. Part B 26 (2006) 526-529.

[7] J. van der Meulen, W. Dickens, M. Burton, J. Fernandes, O052 Gait characteristics of achondroplasia following lower limb-lengthening, Gait Posture 28 (2008) S36-S37.

[8] R. Baker, J.L. McGinley, M.H. Schwartz, S. Beynon, A. Rozumalski, H.K. Graham, et al., The gait profile score and movement analysis profile, Gait Posture 30 (2009) 265-269.

[9] S. Beynon, J.L. McGinley, F. Dobson, R. Baker, Correlations of the gait profile score and the movement analysis profile relative to clinical judgments, Gait Posture 32 (2010) 129-132.

[10] R. Baker, J.L. McGinley, M. Schwartz, P. Thomason, J. Rodda, H.K. Graham, The minimal clinically important difference for the Gait Profile Score, Gait Posture 35 (2012) 612-615.

[11] L. Kark, D. Vickers, A. McIntosh, A. Simmons, Use of gait summary measures with lower limb amputees, Gait Posture 35 (2012) 238-243.

[12] G.M. Johansson, G.E. Frykberg, H. Grip, E.W. Broström, C.K. Häger, Assessment of arm movements during gait in stroke: the arm posture score, Gait Posture 40 (2014) 549-555.

[13] K. Schweizer, J. Romkes, M. Coslovsky, R. Brunner, The influence of muscle strength on the gait profile score (GPS) across different patients, Gait Posture 39 (2014) 80-85.

[14] M.H. Schwartz, A. Rozumalski, The Gait Deviation Index: a new comprehensive index of gait pathology, Gait Posture 28 (2008) 351-357.

[15] S. Armijo-Olivo, S. Warren, J. Fuentes, D.J. Magee, Clinical relevance vs. Statistical significance: using neck outcomes in patients with temporomandibular disorders as an example, Man. Ther. 16 (2011) 563-572.

[16] R.B. Davis, S. Ounpuu, D. Tyburski, J.R. Gage, A gait analysis data collection and reduction technique, Hum. Mov. Sci. 10 (1991) 575-587.

[17] W.T. Dempster, Space requirements of the seated operator: geometrical, kinematic, and mechanical aspects of the body, with special reference to the limbs. (1955).

[18] E. Broström, E.M. Gutierrez-Farewik, M. Örtqvist, L. Hagenäs, L. Neumeyer, A. Rozumalski, et al., A comparison of functional and regression-based hip joint centers in persons with achondroplasia, Gait Posture 30 (2009) S81-S82.

[19] R.M. Ehrig, W.R. Taylor, G.N. Duda, M.O. Heller, A survey of formal methods for determining the centre of rotation of ball joints, J. Biomech. 39 (2006) 2798-2809.

[20] R. Baker, L. Finney, J. Orr, A new approach to determine the hip rotation profile from clinical gait analysis data, Hum. Mov. Sci. 18 (1999) 655-667.

[21] A. Brunner, A.T. Hamers, M. Fitze, R.F. Herzog, The plain $\beta$-angle measured on radiographs in the assessment of femoroacetabular impingement, J. Bone Jt. Surg. 92 (2010) 1203-1208 British.

[22] M. De Pellegrin, D. Moharamzadeh, Ultrasound hip evaluation in achondroplasia, J. Pediatr. Orthop. Part B 28 (2008).

[23] D.T. Sims, G.L. Onambele-Pearson, A. Burden, C. Payton, C.I. Morse, Specific force of the vastus lateralis in adults with Achondroplasia, J. Appl. Physiol. 124 (2018) 696-703.

[24] R.J. Maughan, J.S. Watson, J. Weir, Strength and cross-sectional area of human skeletal muscle, J. Physiol. 338 (1983) 37-49.

[25] S.A. Bruce, S.K. Phillips, R.C. Woledge, Interpreting the relation between force and cross-sectional area in human muscle, Med. Sci. Sports Exerc. 29 (1997) 677-683.

[26] M.E. Harrington, A.B. Zavatsky, S.E.M. Lawson, Z. Yuan, T.N. Theologis, Prediction of the hip joint centre in adults, children, and patients with cerebral palsy based on magnetic resonance imaging, J. Biomech. 40 (2007) 595-602.

[27] R. Hara, J. McGinley, C. Briggs, R. Baker, M. Sangeux, Predicting the location of the hip joint centres, impact of age group and sex, Sci. Rep. 6 (2016) 1-9.

[28] M. Galli, V. Cimolin, C. Rigoldi, A. Kleiner, C. Condoluci, G. Albertini, Use of the gait profile score for the quantification of gait pattern in down syndrome, J. Dev. Phys. Disabil. 27 (2015) 609-615. 